\title{
A comparison of the value of viscosity for several water models using Poiseuille flow in a nano channel
}

\author{
A. P. Markesteijn ${ }^{1}$, Remco Hartkamp ${ }^{2}$, S. Luding ${ }^{2}$, and J. Westerweel ${ }^{1}$ \\ ${ }^{1}$ Laboratory for Aero \& Hydrodynamics, Delft University of Technology, \\ Leeghwaterstraat 21, 2628 CA Delft, The Netherlands and \\ ${ }^{2}$ Multi Scale Mechanics, MESA+ Institute for Nanotechnology, \\ University of Twente, P.O.Box 217, 7500 AE Enschede, The Netherlands
}

(Dated: 8th February 2012)

\begin{abstract}
The viscosity-temperature relation is determined for the water models SPC/E, TIP4P, TIP4P/Ew, and TIP4P/2005 by considering Poiseuille flow inside a nano channel using molecular dynamics. The viscosity is determined by fitting the resulting velocity profile (away from the walls) to the continuum solution for a Newtonian fluid and then compared to experimental values. The results show that the TIP4P/2005 model gives the best prediction of the viscosity for the complete range of temperatures for liquid water, and thus it is the preferred water model of these considered here for simulations where the magnitude of viscosity is crucial. On the other hand, with the TIP4P model the viscosity is severely underpredicted, and overall the model performed worst, whereas, the SPC/E and TIP4P/Ew models perform moderately.
\end{abstract}




\section{INTRODUCTION}

Viscosity plays an important role in many physical transport processes, and hence it is important to specify it accurately in computer simulations in which these processes are investigated. Of special interest is water. When simulating water with molecular dynamics (MD) it is vital to select a water model that correctly predicts the process investigated. Many water models have been developed, differing in parameter values and number of charge sites, and each having different success in predicting the correct value of a certain physical parameter. However, only few references give a complete set of the viscosity versus temperature of a certain water model, which makes it difficult to select the appropriate water model. In this study, the viscosity-temperature relation of four water models is reported. These are the popular 3-point charge SPC/E water model [1], and several variants of the 4-point charge models; TIP4P [2], TIP4P/Ew [3], and the recent TIP4P/2005 [4].

There are several ways to obtain the values of the viscosity by means of MD simulations and most of them require a long simulation time in order to obtain statistically meaningful results. The most frequently used methods are the Green-Kubo method [5, 6] and the Stokes-Einstein method [7]. Both methods are based on the auto-correlation function of the stress tensor, which is computationally expensive to obtain. Furthermore, there are problems in interpretation of the Green-Kubo expressions of the transport coefficients $[8,9]$. Another way of finding the viscosity is by simulating Couette shear flow, i.e. computation of the ratio of shear stress and strain rate. The quality of viscosity obtained from these methods strongly depends on the accuracy of the stress, which in turn strongly depends on the used cut-off radius and method used to compute the long-range interactions [10]. Alternatively, the periodic perturbation method [11] can be used, where the viscosity can be calculated from a steady-state velocity profile generated by a periodic external force applied to the system. Compared to the stress, the velocity profile is straightforward to extract from a MD simulation and requires fewer data points to be collected, i.e. less simulation time, in order to obtain statistically meaningful results.

Previous calculations of the viscosity for different water models showed that there can be a large difference between the calculated value and the experimental value, as discussed next. Most research concentrated on the SPC/E water model. For example Balasubramanian et al. [12] showed how the viscosity of SPC/E is calculated for $T=303.15 K$, using both equilibrium and non-equilibrium molecular dynamics. The value they found is about $18 \%$ less than the experimental value, which is similar to the error that Gou et al. [13] found. Hess et al. [10] used periodic shear flow to calculate the viscosity of the SPC/E water model at $T=300 K$ and found a value about $30 \%$ lower than the experimental value. 
Recently, this has also been verified by Chen et al. [14] using the Green-Kubo method. Wu et al. [15] simulated shear flow at $T=298.5 \mathrm{~K}$ and found an error of about $23 \%$ for SPC/E. On the other hand, Bordat et al. [16], using a reverse non-equilibrium molecular dynamics simulation, calculated the value of the viscosity for the SPC/E water model at $T=300 K$, which was almost the same as the experimental value (within $5 \%$ error).

Less research is done on the other water models. For example, Yongli et al. [17] calculated the value of the viscosity at several liquid water temperatures for several water models (including the TIP4P model) using the Stokes-Einstein relation and reported errors between $30.3 \%$ and $52.3 \%$ between experimental values and calculated values. Wensink et al. [18] calculated the value of the viscosity for the TIP4P model at $T=298.25 \mathrm{~K}$ and found an error of approximately $48 \%$ compared to the experimental data. Very recently, Song et al. [19] performed non-equilibrium molecular dynamics simulations using the periodic perturbation method to simulate the shear viscosity of five commonly used water models (including the SPC/E and TIP4P model). The value they found for the viscosity of the SPC/E model at $T=300 K$ is $15 \%$ less than the experimental value, while the value for the TIP4P model was $41 \%$ less than the experimental value. For the TIP4P/Ew water model no data for the viscosity is known to us, while for the TIP4P/2005 only very recently [20] the dependency of the value of the viscosity on the pressure at three different temperatures were calculated using the Green-Kubo method. The conclusion was that, at least at these temperatures, the value of the viscosity is very well predicted (slightly less than 5\% error) with the TIP4P/2005 water model. Guevara-Carrion et al. [21] compared the SPC, SPC/E, TIP4P and TIP4P/2005 model for the prediction of several transport properties of pure liquid water and mixtures with methanol and ethanol. They found that the TIP4P/2005 model performed better than the other models over the whole range of properties. However, they note that the TIP4P/2005 model does not predict the properties of the saturated vapor phase correctly. Furthermore, in other recent papers [22-24] it was shown how the TIP4P/2005 water model predicts also other material properties with high accuracy and therefore is a promising water model.

In this study, several of the water models (SPC/E, TIP4P, TIP4P/Ew, and TIP4P/2005) are tested on their ability to model the viscosity of liquid water between the temperatures $T=273 \mathrm{~K}$ and $373 \mathrm{~K}$. Especially, it is shown how Poiseuille flow generated inside a nano-sized channel can be used to extract the values of viscosity versus temperature for these models very efficiently. The flow is generated by a constant body force on each of the water molecules inside the channel and the resulting velocity profile is used to calculate the viscosity. This technique was used before to investigate the viscosity of simple 


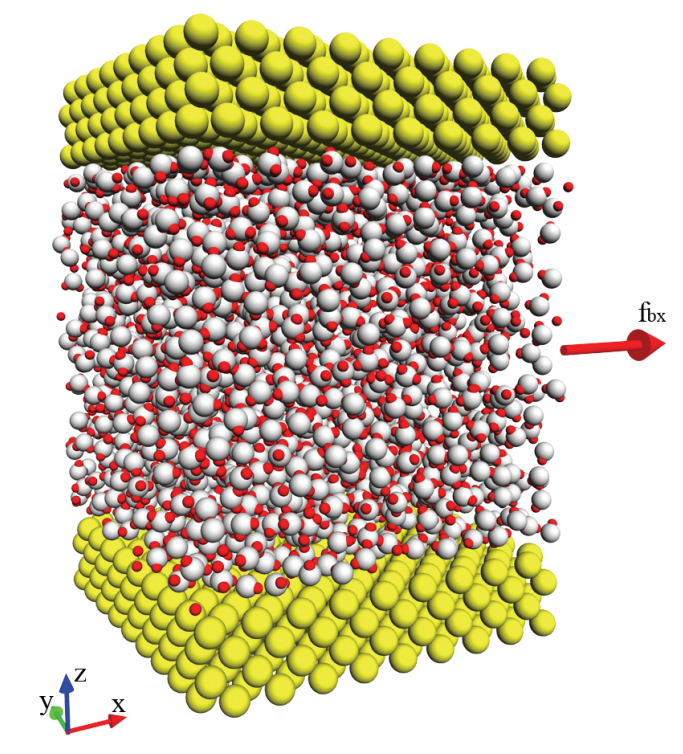

Figure 1: The MD model of the nano channel. In total 2048 water molecules are placed between two solid atomistic walls each consisting of 648 silicon atoms in four layers. The distance between the centres of the two walls is $\approx 4.3$ nm. Poiseuille flow is generated by a body force $f_{b x}$ in the $\mathrm{x}$-direction.

fluids, like argon [25], and has several benefits compared to the other methods. For example, the reported simulation times needed in order to obtain sufficiently converged statistics to calculate the viscosity using the Green-Kubo method, the Stokes-Einstein method, or the Couette shear flow method are 10, 20, or $60 \mathrm{~ns}$, respectively. Water simulations commonly use a time step of 1 or $2 \mathrm{fs}$, meaning that several tens of million time steps are required in these simulations and therefore imply a considerable computational effort. Although the periodic perturbation method performs better, where only $2-4$ ns of simulation time are needed to obtain the results, good statistics for the velocity profile can be obtained within only $1.2 \mathrm{~ns}$ of steady state flow. However, similar to the periodic perturbation method, the viscosity is not obtained in a shear-free situation. Therefore, care must be taken that the shear inside the nano channel does not become too strong.

\section{METHODS AND SIMULATION DETAILS}

\section{A. Poiseuille flow in a nano-sized channel}

The value of the viscosity is calculated for the different water models by examining Poiseuille flow inside a nano-sized channel illustrated in Figure 1. The Poiseuille flow is generated by a constant body force $f_{b x}$ in the $\mathrm{x}$-direction, on each molecule. The channel itself is created by modelling two parallel 
solid atomistic lattice walls at a certain distance from each other in the z-direction. Between the two walls, the water molecules are placed. The boundary conditions in the $\mathrm{x}$ and $\mathrm{y}$-direction are periodic, while in the z-direction the water molecules are constricted by the walls. The equation for (Poiseuille) flow inside a channel in this case is:

$$
\frac{d}{d z}\left(\mu \frac{d u_{x}}{d z}\right)=-\rho f_{b x}
$$

where $u_{x}$ is the (macroscopic) velocity in the $\mathrm{x}$-direction inside the channel, $\rho$ is the liquid density, and $\mu$ is the unknown viscosity. The velocity profile and density can be extracted from the MD simulation, while the applied force is known. This gives the possibility to relate the resulting Poiseuille flow to the viscosity of the used water model [26].

However, care must be taken, since for example, Bitsanis et al. [27] showed that, at least for simple liquids like argon, the effective viscosity inside the channel can increase considerably in very narrow channels (with a height smaller than 5 molecular diameters). Similarly, Li et al. [28] found experimentally that the viscosity of water in a subnanometer gap can be several orders of magnitude larger than the viscosity of bulk water at the same phase point. One reason for this is the wall-fluid interaction, which results in a layering effect of atoms near the wall and this effect only gradually disappears away from the wall [29]. However, despite this, approximately quadratic velocity profiles can be obtained for simple liquids confined to channels only 10 molecular diameters in height [30]. For more complex liquids, like water simulated in this study, the same is true, as shown below. Furthermore, in Section III, also the effect of the confinement of the liquid, i.e. the height of the channel, on the value of the viscosity is studied.

In order to extract the viscosity from the velocity profile, it is important that only the part of the results are used that show the expected continuum behaviour. To examine this, an equilibrium MD simulation (i.e. without applied flow) is carried out, where the variations near the wall and extent of the variations are studied, especially the density and charge distribution profiles. The density profile should show a region with a constant value, i.e. the expected continuum value, in the middle of the channel, while layering of molecules can be visible near the walls. The charge distribution profile is of interest, because this indirectly gives information about the orientation of the water molecules. However, note that in this study, the wall atoms are deliberately not charged. This means that the well-known phenomenon of the electric double layer [31] is not taken into account and the resulting velocity profile is only due to Poiseuille flow. 

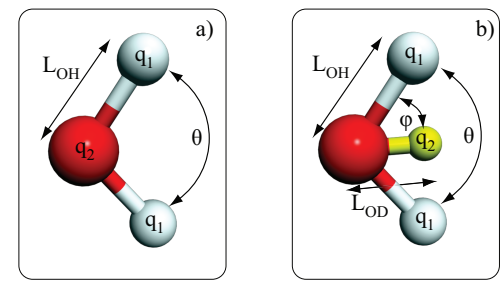

Table I: The parameters of water models for which the value of viscosity is determined. The meaning of the parameters are schematically illustrated in the figure.

\begin{tabular}{ccccc}
\hline & SPC/E & TIP4P & TIP4P/Ew & TIP4P/2005 \\
\hline Type & $\mathrm{a}$ & $\mathrm{b}$ & $\mathrm{b}$ & $\mathrm{b}$ \\
$\epsilon_{O O}(\mathrm{~kJ} / \mathrm{mol})$ & 0.650 & 0.6480 & 0.680946 & 0.7749 \\
$\sigma_{O O}(\AA)$ & 3.166 & 3.15365 & 3.16435 & 3.1589 \\
$q_{1}(e)$ & +0.4238 & +0.5200 & +0.52422 & +0.5564 \\
$q_{2}(e)$ & -0.8476 & -1.0400 & -1.04844 & -1.1128 \\
$L_{O H}(\AA)$ & 1.0000 & 0.9572 & 0.9572 & 0.9572 \\
$L_{O D}(\AA)$ & - & 0.15 & 0.125 & 0.1546 \\
$\theta=\theta_{H O H}\left(^{o}\right)$ & 109.47 & 104.52 & 104.52 & 104.52 \\
$\varphi\left(^{\circ}\right)$ & - & 52.26 & 52.26 & 52.26 \\
\hline
\end{tabular}

\section{B. Water models}

All water models that are investigated in this paper have in common that they solve for the following potential energy equation between molecules $i$ and $j$ :

$$
U=4 \epsilon_{O O}\left[\left(\frac{\sigma_{O O}}{r_{O O}}\right)^{12}-\left(\frac{\sigma_{O O}}{r_{O O}}\right)^{6}\right]+\sum_{\alpha=1}^{N} \sum_{\beta=1}^{N} \frac{q_{i \alpha} q_{j \beta}}{4 \epsilon_{0} r_{i \alpha, j \beta}}
$$

Lennard-Jones interaction between the water molecules is only considered between the O-atoms of each molecule $i$ and $j$, where $r_{O O}$ is the distance between the two atoms. The Lennard-Jones parameters, $\epsilon_{O O}$ and $\sigma_{O O}$ are the interaction energy strength and the distance at which the potential energy is zero, respectively. The Coulombic interaction of the water molecules is computed using a total of $N$ charge sites associated to each water molecule, where $q_{i \alpha}$ is the charge of the $\alpha^{t h}$ charge site of molecule $i$ and $\epsilon_{0}$ is the electrical permittivity of vacuum. The water models investigated in this article use $N=3$ or $N=4$. The bond lengths and angles are fixed using the SHAKE algorithm [32]. The water models differ in the values of the parameters they use. Table I gives an overview of the used parameters in the four models compared here. 


\section{Simulation details}

For each water model, several MD simulations are performed in a (canonical) NVT ensemble. The temperature in the system is controlled by a Nose-Hoover thermostat $[33,34]$ and the simulated temperatures range from 273 to $373 \mathrm{~K}$. The walls of the nano channel are placed approximately $4.3 \mathrm{~nm}$ apart, measured from the centre of the bottom wall lattice to the centre of the top wall lattice.

Each of the atomistic walls consists of four layers of solid atoms, placed and fixed in a fcc lattice, i.e. in a so-called $\mathrm{ABAB}$ stacking. The chosen material properties of the wall are based on silicon, which has a density of $\rho_{\text {wall }}=2329 \mathrm{~kg} / \mathrm{m}^{3}$ at the initialisation temperature of the MD system, $T_{\text {init }}=293$ K. Each wall consists of 648 wall atoms, which interact (only) with the oxygen atoms according to the Lennard-Jones 12-6 potential. The Lennard-Jones parameters for the wall in the case of Silicon are: $\sigma_{\text {wall }-O}=\sigma_{S i-O}=3.24 \AA$ and $\epsilon_{\text {wall }-O}=\epsilon_{S i-O}=1.274 \mathrm{~kJ} / \mathrm{mol}$ [35]. These values are the initial $/$ standard values and are used in all simulations, unless stated otherwise. The total number of water molecules between the two walls is 2048. These are also initialised in a fcc lattice, with an initial density of $\rho_{\text {init }}=998.2 \mathrm{~kg} / \mathrm{m}^{3}$, and are allowed to melt during the equilibration process [36], which takes $0.3 \mathrm{~ns}$.

The value of the body force $f_{b x}$ is chosen such that the typical maximum velocity inside the channel never exceeds $20 \mathrm{~m} / \mathrm{s}$. This was done to prevent the shear rate inside the nano channel becoming too large. This maximum velocity is selected since it provides a high signal-to-noise ratio. It was verified that the value of the viscosity was not greatly affected by the amount of generated shear in the channel by performing simulations with different values of $f_{b x}$.

The integration of Newton's equation of motion is performed with the Verlet algorithm [37], while the electrostatic interactions are treated by the 'Particle-Particle and Particle-Mesh' (PPPM) method [38]. However, the MD domain only has periodic boundary conditions specified in two dimensions, the PPPMmethod must be adapted to prevent erroneous summation in the direction perpendicular to the walls. The method adopted here is the 2D-Slab method with an added (vacuum) space at both sides of the wall [39], and includes the ELC term to prevent slab-slab interactions [40, 41]. The grid size for the PPPM method is $[36 \times 36 \times 60]$ with a splitting parameter $\beta=0.305$, while the cut-off value for the Lennard-Jones interaction is $r_{c}=1.0 \mathrm{~nm}$, which is approximately $3 \sigma_{O O}$. In order to verify whether the cut-off radius and the used grid size for the PPPM method are sufficient for obtaining the (correct) velocity profile, several simulations are performed using different quantities. This effectively controls the accuracy in obtaining the Lennard-Jones interactions and the electrostatic interactions. The results will show to what degree the velocity profile is influenced by this change. 

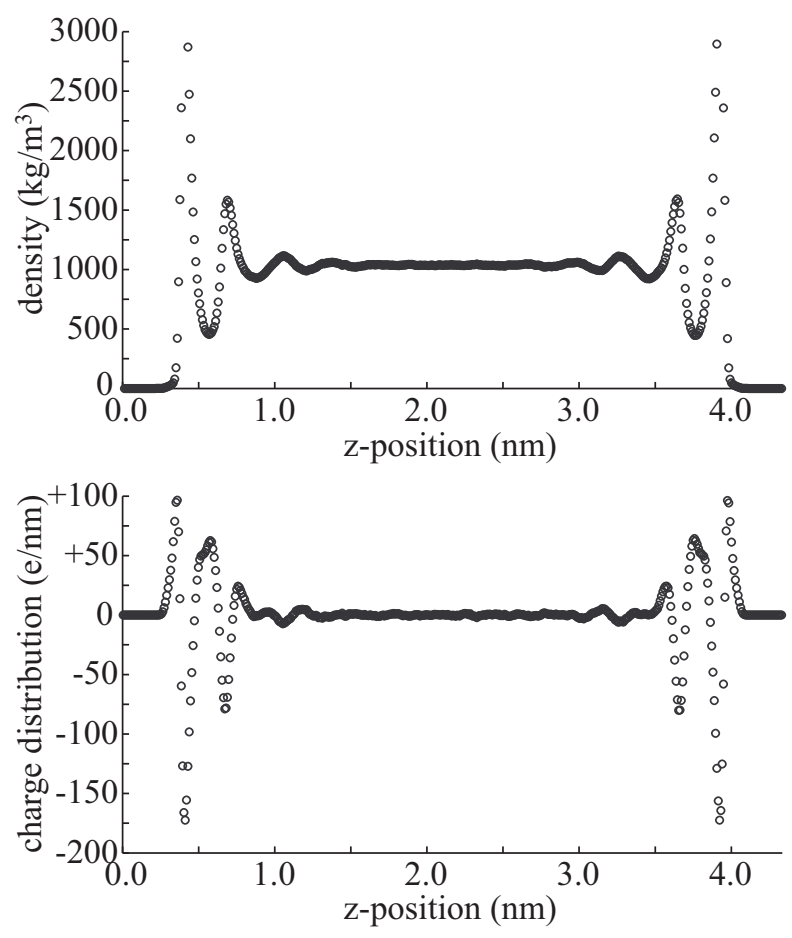

Figure 2: The density profile (top) and charge distribution profile (bottom) obtained for the nano channel from the equilibrium MD simulation ( $\rho_{\text {init }}=998.2 \mathrm{~kg} / \mathrm{m}^{3}, T_{\text {init }}=293 \mathrm{~K}$, using the SPC/E water model)

For each MD simulation, the total simulation time is $1.5 \mathrm{~ns}$, with a MD time step of 1.0 fs. All results are determined from the last $1.2 \mathrm{~ns}$ of the simulation and are obtained by binning the different macroscopic values in 500 bins, which are equally distributed across the z-direction. This relatively small simulation time was verified to be sufficient to obtain consistent values for the viscosity. A test simulation with a total simulation time of $4.2 \mathrm{~ns}$ was compared to the shorter simulation. The calculated value of viscosity did not change more than $1 \%$ and the statistical error of the data from the longer simulation decreased slightly.

\section{RESULTS AND DISCUSSION}

\section{A. Variations near the channel walls}

First the equilibrium MD simulation is carried out in order to study the variations near the wall. The water model SPC/E is employed for this simulation, for which the parameters can be found in table I. During the simulation, the density and charge distribution values are collected in the bins.

Figure 2 (top) shows the density profile while the bottom figure shows the charge distribution across the channel. The density profile shows large variations near the wall around a more or less constant value 
in the middle of the channel. These variations are the result of the interaction of the water molecules with the solid atoms of the wall and can also be observed experimentally [42]. Water molecules spend more time inside certain layers parallel to the walls, where the interaction between the wall and the surrounding liquid is closer to equilibrium than elsewhere. These layers are situated at the peaks visible in the density profile and come from the fact that, due to the strong LJ repulsion, O-atoms have a minimal distance in $\mathrm{z}$-direction, but can be disordered (inside the layer) in $\mathrm{x}-\mathrm{y}$ direction. On the other hand, because of the dense layer of water molecules, the remaining molecules rarely come too close to this layer because of the strong repulsion at close range. On either side of a dense layer, fewer molecules are present on average, which are the troughs in the density profile. Only when the water molecules are far enough from the wall, the interaction between the surrounding water molecules becomes isotropic due to increased disorder, and the result is that more or less constant density is reached.

The charge distribution profile shows that the orientation of the water molecules is also constricted near the walls. The first strong positive peak of the profile indicates that more $\mathrm{H}$-atoms than $\mathrm{O}$-atoms can be found near the wall, while the first trough after the peak shows a strong negative charge indicating a layer of mostly $\mathrm{O}$-atoms coinciding with the first peak of the density profile. The second peak and trough show something similar. On the other hand, in the middle of the channel the average charge is zero, indicating random distribution and orientation of the water molecules. Note that the total charge averaged across the entire profile equals zero, e.g. the water inside the channel is neutral. Although in the simulation the electrokinetic effect caused by charges of the wall and counter-ions in the water, i.e. the electric double layer, was deliberately not simulated, the orientation of the water molecules itself can create a charge distribution in a direction normal to the wall.

Near-bulk or continuum conditions are established $1.2 \mathrm{~nm}$ away from the walls, indicating the possibility to extract the value of the viscosity. However, the value of the density in the middle of the channel is about $1035 \mathrm{~kg} / \mathrm{m}^{3}$, while the averaged density across the complete density profile is exactly the same as the initialised value, $\rho_{\text {init }}=998.2 \mathrm{~kg} / \mathrm{m}^{3}$. The reason for the higher value of density measured in the middle of the channel is the combination of the Lennard-Jones parameters for the wall-fluid interaction and the resulting interaction with the water molecules.

In order to compare the value of the viscosity of each water model to the experimental value of the viscosity, each simulation should be performed with a predefined value of the density in the middle of the channel. The value of the density that is aimed for, is the value at 1 bar for the different temperatures, i.e. $\left.\rho(T)\right|_{p=c o n s t}$ and can be found for example in the book of Bird et al. [43]. There are several ways how 
this can be accomplished. One possibility is to change the distance between the two walls of the channel accordingly. This is comparable to what is done in an NPT-simulation, where the pressure is kept constant by changing the size of the MD simulation box, i.e. effectively changing the volume. Another possibility is to change the number of water molecules between the walls, i.e. effectively changing the mass of fluid. However, we chose to keep the volume and mass constant and used an alternative method to change the bulk density. Namely, the correct density in the middle of the channel is obtained by adjustment of the wall-fluid interaction parameter of the wall, $\sigma_{\text {wall }-O}$. If this value is decreased, water molecules are able to move closer to the wall and therefore, on average, have access to a larger volume between the two walls. This results in a lower density in the middle of the channel. The following simulations are therefore performed with a variable value of the wall-fluid interaction parameter $\sigma_{\text {wall-O}}$. The (macroscopic) density can easily be sampled and does not require a long simulation time before a meaningful value is obtained. Therefore, the correct value of $\sigma_{\text {wall-O}}$ can be obtained within the equilibration process (i.e. within $0.3 \mathrm{~ns}$ of simulation time) and the remainder of the simulation is performed with this obtained value. Typical values of $\sigma_{\text {wall-O }}$ resulting from such a simulation ranged from 0.82 to 1.05 times the nominal value.

\section{B. Viscosity of the water models}

The viscosity will be determined for the four different water models, SPC/E, TIP4P, TIP4P/Ew and TIP4P/2005 for a temperature range of $T=273 K-373 K$ by simulating Poiseuille flow in the nano channel. As noted before, the value of the wall-fluid interaction parameter of the wall, $\sigma_{\text {wall-O}}$ is changed such that the density in the middle of the channel is equal to the (experimental) value of the density of water at a pressure of 1 bar. Furthermore, the interaction strength between the wall and the water molecules is changed/increased to: $\epsilon_{\text {wall }-O}=3 \epsilon_{S i-O}=3.822^{\mathrm{kJ}} / \mathrm{mol}$. This was done in order to reduce any significant slip developing near the wall.

Figure 3 shows a typical velocity profile obtained from such a simulation. As expected, the velocity profile is similar to a Poiseuille flow velocity profile, with only minor differences very near the wall. In order to determine the viscosity from the velocity profile, equation II. 1 is rewritten to:

$$
\mu=\frac{-\rho f_{b x}}{d^{2} u_{x} / d z^{2}}
$$

Effectively, this means that the second derivative of the velocity profile needs to be measured and the easiest way to do this is to curve fit the data points of the velocity profile from the MD simulation with 


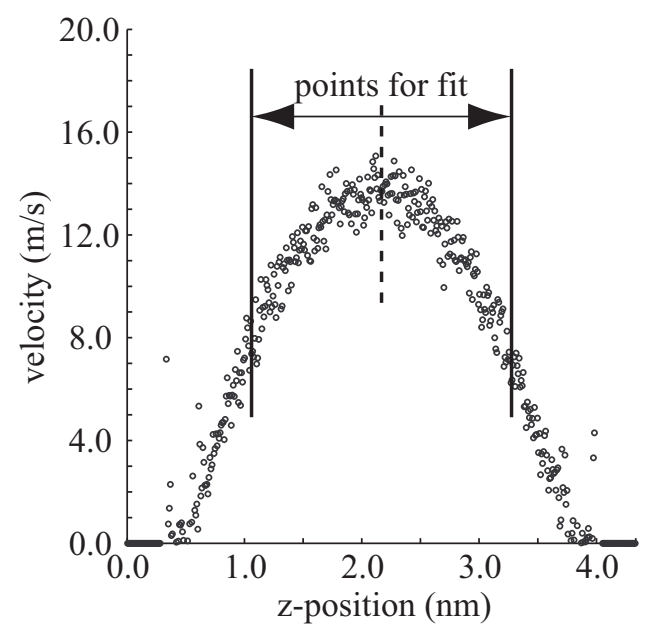

Figure 3: A typical velocity profile from one of the simulations of water inside a nanochannel (using TIP4P/2005). An illustration of the fraction of the velocity profile used for the curve fit is given.

Table II: The values of viscosity from the four water models as obtained from the fitted velocity profile and the errors in percent between the calculated and experimental values of the viscosity of water.

\begin{tabular}{|c|c|c|c|c|c|c|c|c|c|}
\hline \multirow[t]{2}{*}{$\mathbf{T}(\mathbf{K})$} & \multicolumn{2}{|l|}{ SPC/E } & \multicolumn{2}{|l|}{ TIP4P } & \multicolumn{2}{|c|}{ TIP4P/Ew } & \multicolumn{2}{|c|}{ TIP4P/2005 } & \multirow{2}{*}{$\begin{array}{l}\text { Experiment } \\
\mu_{\exp }(\mathrm{mPas}) \\
\end{array}$} \\
\hline & $\mu(\mathrm{mPas})$ & $\frac{\left(\mu-\mu_{\exp }\right)}{\mu_{\exp }}$ & $\mu(\mathrm{mPas})$ & $\frac{\left(\mu-\mu_{\exp }\right)}{\mu_{\exp }}$ & $\mu(\mathrm{mPas})$ & $\frac{\left(\mu-\mu_{\exp }\right)}{\mu_{\exp }}$ & $\mu(\mathrm{mPas})$ & $\frac{\left(\mu-\mu_{\exp }\right)}{\mu_{\exp }}$ & \\
\hline 273 & $1.282 \pm 0.0940$ & $-27.9 \%$ & $0.668 \pm 0.0515$ & $-62.4 \%$ & $1.601 \pm 0.1459$ & $-10.0 \%$ & $1.697 \pm 0.1259$ & $-4.6 \%$ & 1.778 \\
\hline 277 & $1.073 \pm 0.0556$ & $-31.7 \%$ & $0.698 \pm 0.0232$ & $-55.6 \%$ & $1.196 \pm 0.0776$ & $-23.9 \%$ & $1.506 \pm 0.1125$ & $-4.2 \%$ & 1.572 \\
\hline 283 & $0.879 \pm 0.0356$ & $-32.6 \%$ & $0.605 \pm 0.0179$ & $-53.6 \%$ & & $-18.9 \%$ & & $-14.5 \%$ & 1.303 \\
\hline 293 & $0.795 \pm 0.0473$ & $-20.8 \%$ & $0.544 \pm 0.0143$ & $-45.8 \%$ & $0.744 \pm 0.0261$ & $-25.9 \%$ & $0.928 \pm 0.0341$ & $-7.6 \%$ & 1.004 \\
\hline 303 & $0.663 \pm 0.0239$ & $-17.3 \%$ & $0.479 \pm 0.0146$ & $-40.2 \%$ & $0.705 \pm 0.0249$ & $-12.0 \%$ & $0.817 \pm 0.0476$ & $+1.9 \%$ & 0.802 \\
\hline 313 & $0.519 \pm 0.0134$ & $-21.1 \%$ & $0.402 \pm 0.0089$ & $-38.8 \%$ & $0.538 \pm 0.0148$ & $-18.2 \%$ & $0.586 \pm 0.0280$ & $-10.8 \%$ & 0.658 \\
\hline 323 & $0.424 \pm 0.0154$ & $-23.1 \%$ & $0.325 \pm 0.0125$ & $-41.0 \%$ & $0.483 \pm 0.0240$ & $-12.3 \%$ & $0.557 \pm 0.0248$ & $+1.0 \%$ & 0.551 \\
\hline 343 & $0.370 \pm 0.0193$ & $-8.9 \%$ & $0.285 \pm 0.0135$ & $-29.9 \%$ & $0.384 \pm 0.0218$ & $-5.5 \%$ & $0.408 \pm 0.0152$ & $+0.3 \%$ & 0.407 \\
\hline 363 & $0.301 \pm 0.0097$ & $-4.2 \%$ & $0.280 \pm 0.0159$ & $-11.1 \%$ & $0.270 \pm 0.0110$ & $-14.3 \%$ & $0.320 \pm 0.0135$ & $+1.8 \%$ & 0.315 \\
\hline 373 & $0.271 \pm 0.0068$ & $-3.6 \%$ & $0.251 \pm 0.0128$ & $-10.5 \%$ & $0.264 \pm 0.0110$ & $-6.1 \%$ & $0.291 \pm 0.0120$ & $+3.8 \%$ & 0.281 \\
\hline
\end{tabular}

a parabola. From this fitted velocity profile, the (analytical) second derivative with respect to the height of the channel can be taken easily. In detail, the data points, e.g. as shown in Figure 3, are fitted to a function described by $u=u_{0}+a\left(z-z_{0}\right)^{2}$, where the fitting parameters $u_{0}$ and $a$ need to be determined and the parameter $z_{0}$ is taken as the middle of the channel. Doing so, means the obtained velocity profile is assumed to be symmetric, while the fitting parameter $a$ is directly related to the second derivative and 
therefore the viscosity by: $\mu=-\rho f_{b x} / 2 a$. However, because this value is sensitive to the actual fit, multiple symmetric curve fits of different (continuum-like) sections of the (same) velocity profile of the MD results are taken. The sections of the velocity profile that are used for the fit, are described by the fraction of the total velocity profile, where the value of 1.0 means the obtained velocity profile from wall to wall is used. In total 10 fits for each MD velocity profile are performed. The fractions for the set of curve fits are: $0.80,0.75,0.70,0.65,0.60,0.55,0.50,0.45,0.40$, and 0.35 . This means that for each simulation a set of ten values of $a$ is obtained including ten fit errors. Therefore, the final value for the viscosity is obtained by averaging the resulting set of fits and the error of the fitted value is estimated using the corresponding $95 \%$ confidence bounds of the set of fits. Therefore, note that the error described in the results is the fitting error of the curve rather than the statistical error resulting from the MD simulation.

However, before the results of the value of viscosity for several water models are discussed, first the method is compared to results obtained using different methods. As mentioned in the introduction, the method to obtain the viscosity using the velocity profile in a MD simulation was used by Koplik et al. [25] for argon. The value of viscosity they computed was very similar to the value obtained using other methods, while the cut-off radius they used was (only) $2.5 \sigma$. It is expected that in the case when water is simulated, the same method to determine the viscosity can be used, however first it must be determined whether the velocity profile, and therefore the computed value of the viscosity, is not highly influenced by simulation parameters like the confinement of the water molecules between the two walls, the used cut-off radius, and the accuracy of the evaluation of the electrostatic interactions. To test this, four independent simulations are performed where one of the parameters is changed and the velocity profile and viscosity are compared to one reference simulation. The reference simulation uses the simulation parameters as specified in Section II C.

All these simulations are done with the TIP4P water model at $T=298.25 \mathrm{~K}$, for which at least three different values of the viscosity can be found in literature. For example, Wensink et al. [18] calculated the value of the viscosity for the TIP4P model at $T=298.25 K$ and found the values $\mu=0.464 \pm$ $0.003 \mathrm{mPas}(48.2 \%$ error) and $\mu=0.479 \pm 0.009 \mathrm{mPas}(46.5 \%$ error $)$ using the periodic perturbation method. Song et al. [19] used the same method and found the values $\mu=0.505 \pm 0.007 \mathrm{mPas}(43.6 \%$ error) and $\mu=0.506 \pm 0.006 \mathrm{mPas}$ (43.5\% error) for the TIP4P model at $T=300 \mathrm{~K}$. Finally, Yongli et al. [17] used the Stokes-Einstein relation and reported only errors, which are between $30.3 \%$ and $52.3 \%$ between experimental values and calculated values. The reference simulation performed using the method described in this article leads to a value for the viscosity: $\mu=0.481 \pm 0.015 \mathrm{mPas}(46.3 \%$ 
error) and therefore similar to the results obtained by others using different methods. In order to verify the sensitivity of several model parameters on the viscosity, the four other simulations are compared to this value.

The first simulation compares the results of the reference simulation to the results obtained from a simulation where the distance of the walls is $\approx 6.4 \mathrm{~nm}$ which is 1.5 times larger than the reference case. This means the number of water molecules inside the channel is $N=3072$ and the fluid is less confined, i.e. a larger part of the density profile does not show variations, compared to the reference case. In order to maintain the same accuracy in obtaining the electric interactions compared to the reference simulation, the PPPM grid size also needs to be increased. In order to do so, the method described by Deserno et al. [44] is used. The resulting PPPM grid size is $[36 \times 36 \times 72]$ with a splitting parameter $\beta=0.306$. The cut-off value for the Lennard-Jones interaction is kept at $r_{c}=1.0 \mathrm{~nm}$. As expected, the resulting density profile of this simulation showed a larger range where the value is the (expected) continuum value, while only near the walls large variations are visible, similar to those in Figure II A. However, the shape of the velocity profile did not change much. The viscosity is calculated from this velocity profile and the resulting value is: $\mu=0.508 \pm 0.032 \mathrm{mPas}$ ( $43.2 \%$ error), which is the range of errors obtained by others previously.

The next three simulations deal with the influence of the used cut-off radius on the velocity profile and the value of viscosity. The first two simulations check whether the PPPM size and therefore the accuracy of the electric interaction has an influence. In order to test this, the following grid sizes are compared: $[18 \times 18 \times 32]$ and $[72 \times 72 \times 120]$, with splitting parameter $\beta=0.253$ and $\beta=0.350$, respectively. This corresponds to a factor 10 decrease and increase in accuracy compared to the reference simulation, respectively. The results from these simulation showed that the computed values of viscosity are: $\mu=0.516 \pm 0.017 \mathrm{mPas}$ (42.4\% error) and $\mu=0.531 \pm 0.018 \mathrm{mPas}$ (40.7\% error), in the case of lower and higher accuracy, respectively. The third simulation tests whether the cut-off radius used for the Lennard-Jones interactions has an influence. This simulation uses a cut-off radius of: $r_{c}=1.2 \mathrm{~nm}$, while the accuracy of the electric interactions remains the same. The results from this simulation leads to a viscosity of: $\mu=0.455 \pm 0.013$ (49.1\% error).

In conclusion, the results show that the calculated values of the viscosity are not highly sensitive to the change of simulation parameters like the PPPM size, cut-off radius, and size of the MD domain. The values of the viscosity that are obtained range from $0.455 \mathrm{mPas}$ to $0.531 \mathrm{mPas}$, which means the error between the experimental and the calculated value is between $40.7 \%$ and $49.1 \%$. This is within the range 


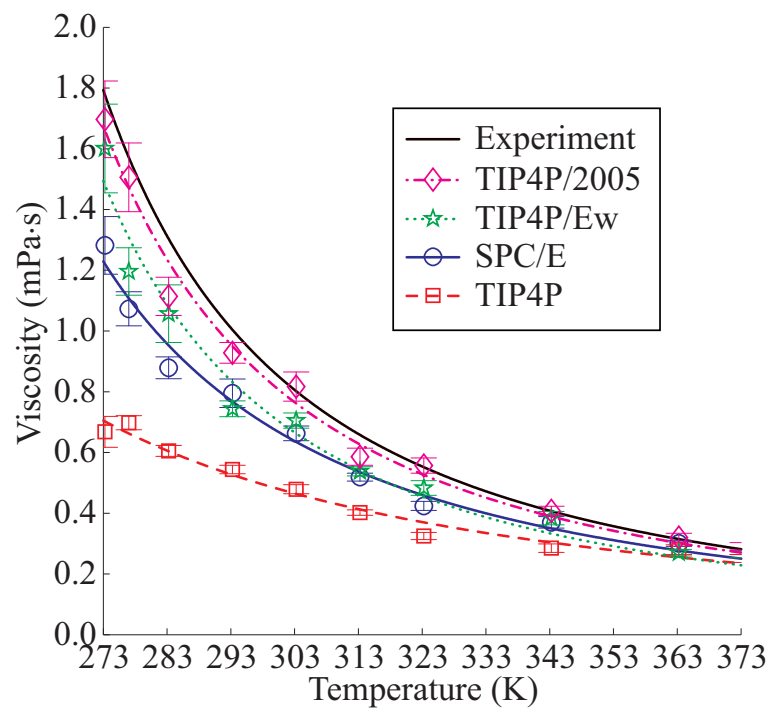

Figure 4: The values of the viscosity as a function of the temperature obtained from the curve fit of the velocity profile for the four different water models. The errors of the fits is also displayed. The lines in the figure are obtained from a fit of the type: $\mu=\left(T-T_{0}\right)^{-b}$, where the fit with experimental data from Bird et al. [43] is used for reference.

Table III: The parameters obtained from the fit of the type $\mu=\left(T-T_{0}\right)^{-b}$ from the data points as shown in figure 4.

\begin{tabular}{c|c|cccc} 
& Experiment & TIP4P/2005 & TIP4P/Ew & SPC/E & TIP4P \\
\hline$T_{o}$ & 225.4 & 224.0 & 224.5 & 212.4 & 173.6 \\
$b$ & 1.637 & 1.642 & 1.677 & 1.633 & 1.578
\end{tabular}

of errors obtained by others using different methods. Further research is necessary in order to examine the full extend of the influence of the simulation parameters on the viscosity. Next the results of the simulations using the four different water models are discussed.

Figure 4 shows the obtained values for the viscosity and the errors of the fits for the four different water models. The experimental values of the viscosity are displayed for reference. The curves are obtained from a fit of the type: $\mu=\left(T-T_{0}\right)^{-b}$, where temperatures are scaled by units Kelvin $(K)$ and $\mu$ is in units of mPas, fitted to experimental data, e.g. from Bird et al. [43], where $T_{0}=225.4 \mathrm{~K}$ and $b=1.637$. Table II shows the obtained values of viscosity from the four different water models and the deviation from the experimental value of viscosity in percent. Table III shows the fit parameters obtained from the simulation results of the four water models.

The results show that the TIP4P water model severely underpredicts the value of the viscosity at all liquid water temperatures, especially at the lower temperatures, where the deviation from the experi- 
mental value is $45 \%$ to $60 \%$, as found also by others $[17,18]$. The SPC/E model and the TIP4P/Ew water model show comparable performance in predicting the value of the viscosity. The TIP4P/Ew water model is slightly better, but does so with more computational effort because of the extra interaction site involved. In general the deviations are about $15 \%$ to $30 \%$ for the SPC/E water model and $10 \%$ to $25 \%$ for the TIP4P/Ew water model at the lower temperatures. The error for the SPC/E water model at $T=293 \mathrm{~K}$ and $T=303 K$ are very similar to the errors reported by others [10, 12-15]. Both models predict the value of the viscosity within $10 \%$ to $15 \%$ for the higher temperatures. However, the TIP4P/2005 water model performed best. In general, the errors for the whole range of liquid water temperatures are below $8 \%$, besides two outliers that are below $15 \%$.

Overall, the averaged data confirm the above conclusions; when the values are averaged over the whole temperature-range, the TIP4P model underpredicts the viscosity by as much as 39\%, the SPC/E and TIP4P/Ew water models underpredict the viscosity by $19 \%$ and 15\%, respectively, while the TIP4P/2005 water model underpredicts the value of viscosity by (only) $3 \%$.

\section{CONCLUSIONS}

The numerical results presented in this paper show how the viscosity for four different water models is calculated and how it compares to experimental values. This was accomplished by simulating Poiseuille flow in a MD nano channel. The value of the viscosity was determined by curve fitting the resulting velocity profile and comparison of the profile to a continuum Newtonian solution. The benefit of using this method is the fact that good statistics for the velocity profile can be obtained within only $1.2 \mathrm{~ns}$ of steady state flow, which is considerably faster than alternative methods for finding the viscosity from MD simulations. The results from the simulations showed that of the four models considered here the TIP4P/2005 water model gives the best prediction of the viscosity over a wide range of temperatures of liquid water, the TIP4P model performs worst, while the SPC/E and TIP4P/Ew water model performed similar and resulted in moderate accuracy for the value of the viscosity. Therefore, if a simulation is required where the viscosity plays an important role, the TIP4P/2005 water model is recommended. Finally, note that in this study, several parameters are varied and the effect of varying these for most of them is small. However, a more detailed parameter study is needed to understand the possibility of a systematic dependency of the viscosity on system and model parameters like, e.g. system size, cut-off radius or wall properties. Furthermore, the models were not evaluated concerning the phase transitions or other more advanced properties of water, neither was the polarisability of water taken into account, so 
that enough options for future research remain.

[1] H. Berendsen, J. Grigera, and T. Straatsma, J. Phys. Chem. 91, 6269 (1987).

[2] W. Jorgensen and J. Madura, Mol. Phys. 56, 1381 (1985).

[3] H. Horn, W. Swope, J. Pitera, J. Madura, T. Dick, G. Hura, and T. Head-Gordon, J. Chem. Phys. 120, 9665 (2004).

[4] J. Abascal and C. Vega, J. Chem. Phys. 123, 234505 (2005).

[5] M. Green, J. Chem. Phys. 22, 398 (1954).

[6] R. Kubo, J. Phys. Soc. Japan 12, 570 (1957).

[7] A. Einstein, Investigations on the Theory of the Brownian Movement (Dover, New York, 1956).

[8] R. García-Rojo, S. Luding, and J. Brey, Phys. Rev. E 74 (2006).

[9] V. Kumaran, Phys. Rev. Lett. 96 (2006).

[10] B. Hess, J. Chem. Phys. 116, 209 (2002).

[11] E. Gosling, I. McDonald, and K. Singer, Mol. Phys. 26, 1475 (1973).

[12] S. Balasubramanian, C. Mundy, and M. Klein, J. Chem. Phys. 105, 11190 (1996).

[13] G. Guo and Y. Zhang, Mol. Phys. 99, 283 (2001).

[14] T. Chen, B. Smit, and A. Bell, J. Chem. Phys. 131, 246101 (2009).

[15] Y. Wu, H. Tepper, and G. Voth, J. Chem. Phys. 124, 024503 (2006).

[16] P. Bordat and F. Müller-Plathe, J. Chem. Phys. 116, 3362 (2002).

[17] S. Yongli, S. Minhua, C. Weidong, M. Congxiao, and L. Fang, Comp. Mat. Science 38, 737 (2007).

[18] E. Wensink, A. Hoffmann, P. van Maaren, and D. van der Spoel, J. Chem. Phys. 119, 7308 (2003).

[19] Y. Song and L. Dai, Mol. Sim. 36, 560 (2010).

[20] M. Gonzàlez and J. Abascal, J. Chem. Phys. 132, 096101 (2010).

[21] G. Guevara-Carrion, J. Vrabec, and H. Hasse, J. Chem. Phys. 134, 074508 (2011).

[22] J. Alejandre and G. Chapela, J. Chem. Phys. 132, 014701 (2010).

[23] J. Abascal, E. Sanz, and C. Vega, Phys. Chem. Chem. Phys. 11, 556 (2009).

[24] H. Pi, J. Aragones, C. Vega, E. Noya, J. Abascal, M. Gonzalez, and C. McBride, Mol. Phys. 107, 365 (2009).

[25] J. Koplik, J. Banavar, and J. Willemsen, Phys. Rev. Lett. 60, 1282 (1988).

[26] B. Todd and D. Evans, J. Chem. Phys. 103, 9804 (1995). 
[27] I. Bitsanis, S. Somers, H. Davis, and M. Tirell, J. Chem. Phys. 93, 3427 (1990).

[28] T. Li, J. Gao, R. Szoszkiewicz, U. Landman, and E. Riedo, Phys. Rev. B 75, 115415 (2007).

[29] R. Hartkamp and S. Luding, in International Conference on Multiphase Flow. Tampa, Florida, May 2010 (2010).

[30] K. Travis, B. Todd, and D. Evans, Phys. Rev. E 55, 4288 (1997).

[31] J. Lyklema, Fundamentals of Interface and Colloid Science, Volume II (Academic Press Limited, 1995).

[32] J. Ryckaert, G. Ciccotti, and H. Berendsen, J. Comp. Phys. 23, 327 (1977).

[33] S. Nosé, J. Chem. Phys. 81, 511 (1984).

[34] W. Hoover, Phys. Rev. A 31, 1695 (1985).

[35] G. Karniadakis, A. Beskok, and N. Aluru, Microflows and nanoflows: fundamentals and simulation (Springer, 2005).

[36] D. Frenkel and B. Smit, Understanding Molecular Simulations: From Algorithms to Applications, 2nd ed. (Academic Press, 2002).

[37] L. Verlet, Phys. Rev. 159 (1967).

[38] R. Hockney and J. Eastwood, Computer Simulation Using Particles (IOP, 1988).

[39] E. Spohr, J. Chem. Phys. 107, 6342 (1997).

[40] A. Arnold, J. de Joannis, and C. Holm, J. Chem. Phys. 117, 2496 (2002).

[41] J. de Joannis, A. Arnold, and C. Holm, J. Chem. Phys. 117, 2503 (2002).

[42] D. Chan and R. Horn, J. Chem. Phys. 83, 5311 (1985).

[43] R. Bird, W. Stewart, and E. Lightfoot, Transport Phenomena, 2nd ed. (Wiley, 2002).

[44] M. Deserno and C. Holm, J. Chem. Phys. 109, 7694 (1998). 